\title{
画HAD
}

ISSN-L: 2530-5115

DOI: http://doi.org/10.22585/hospdomic.v3i2.72

\section{Acceso abierto, ciencia abierta y Plan S: su posible repercusión}

\section{Open access, open science and Plan S: its possiblerepercussion}

Cristina A. Fraga Medín', Cristina Bojo Canales'.

1. Biblioteca Nacional de Ciencias de la Salud. Instituto de Salud Carlos III, Madrid, España.

Correspondencia/Correspondence

Cristina A. Fraga Medín

Biblioteca Nacional de Ciencias de la Salud.

Instituto de Salud Carlos III, Madrid, España.

CRISTINAFRAGA@isciii.es

Recibido/Received

14.04.2019

Aceptado/Accepted

14.04.2019
Conflicto de Intereses/Competing interest Las autoras declaran la inexistencia de conflicto de interés

CÓMO CITAR ESTE TRABAJO | HOW TO CITE THIS PAPER

Fraga Medín CA, Bojo Canales C. Acceso abierto, ciencia abierta y Plan S: su posible repercusión. Hosp Domic. 2019;3(2):163-72. 


\section{INTRODUCCIÓN: ACCESO ABIERTO Y CIENCIA ABIERTA}

El monopolio de las grandes editoriales científicas, que concentran una amplísima mayoría de las revistas y las políticas editoriales cada vez más agresivas en lo económico y en cuanto a las restricciones derivadas del copyright de los artículos publicados en ellas, unido a las posibilidades que ofrecían las nuevas tecnologías y a una disminución muy importante de los costes de edición, publicación y distribución, fueron dando paso, a principios del siglo XXI, a un movimiento que promueve el libre acceso a toda clase de materiales científicos (1).

El movimiento de Open Access tiene sus antecedentes en tres declaraciones que sentaron las bases y principios fundacionales: las Declaraciones de Budapest y Bethesda, en el año 2002 y la Declaración de Berlín en 2003.

La primera de ellas, la Declaración de Budapest (2), impulsada por el Open Society Institute, define el acceso abierto a la literatura científica como:

su disponibilidad gratuita en la internet pública, para que cualquier usuario la pueda leer, descargar, copiar, distribuir, imprimir, con la posibilidad de buscar o enlazar todos los textos de estos artículos, [...] o utilizarlos para cualquiera otro propósito legal, sin barreras financieras, legales o técnicas, distintas de la fundamental de ganar acceso a la propia Internet.

La Declaración de Bethesda (3) completa a la anterior y establece dos de las bases del OA, la reutilización de los contenidos y su disponibilidad a través de repositorios:

el/los autor/es y el/los propietario/s de los derechos de propiedad intelectual otorgan a los usuarios un derecho libre, irrevocable, universal y perpetuo de acceso y licencia para copiar, utilizar, distribuir, transmitir y presentar el trabajo públicamente y hacer y distribuir obras derivadas, en cualquier soporte digital para cualquier finalidad responsable, sujeto a la apropiada atribución de la autoría, así como el derecho de hacer una pequeña cantidad de copias impresas para su uso personal.

Quedaron así establecidos los pilares sobre los que descansa el OA: el acceso libre y gratuito a la información científica y los permisos que tiene el lector/usuario para reutilizar ese trabajo, que podrán ser más o menos restrictivos en función de la licencia escogida para su publicación.

Son dos las vías de implementación de esta forma de difusión del conocimiento científico la vía verde -o archivo en repositorios- y la vía dorada o publicación en acceso abierto.

La vía verde consiste en el autoarchivo -por parte del autor- o archivo delegado -a través de terceros- de la versión final del artículo publicado (versión de editorial), el manuscrito final revisado por pares (versión del autor, sin maquetar por la editorial), o el manuscrito antes de la revisión, en un repositorio. 
La vía dorada consiste en la publicación en revistas científicas de acceso abierto, esto es, revistas que, previo pago, o no, de unas tasas de publicación (los llamados APC, article processing charge) permiten acceso, inmediato o tras un periodo de embargo, a sus contenidos sin ningún coste para el lector y además, establecen los permisos de reutilización de esos trabajos. Estos permisos se establecen mediante licencias que ofrecen un marco jurídicamente válido al acceso abierto. Las licencias más utilizadas en el ámbito académico y de la publicación científica son las Creative Commons (CC). Mediante ellas se ofrecen algunos derechos a los usuarios finales (Ios lectores) bajo ciertas condiciones que son Reconocimiento (Attribution), Uso No Comercial (Non commercial), Sin obras derivadas (No Derivate Works) y Compartir Igual (Share alike).

Durante estos años la generalización de las prácticas de acceso abierto entre los principales actores implicados (investigadores, editores, financiadores) ha sido muy importante. En uno de sus últimos informes, la Comisión Europea (4) cifra en un 74,85\% el porcentaje de artículos en acceso abierto resultantes de proyectos participantes en Horizonte 2020 a finales del año 2017.

De acuerdo con la taxonomía FOSTER, la ciencia abierta es el movimiento para hacer que la investigación científica, los datos y su difusión sean accesibles a una sociedad ávida de conocimiento.

Basada en los principios de transparencia, reutilización, participación, cooperación, responsabilidad y reproducibilidad de la investigación, la prácticas de la ciencia abierta engloban el acceso abierto a las publicaciones de investigación, el intercambio de datos, los open notebooks, la transparencia en la evaluación de la investigación (open peer review), la reproducibilidad de la investigación (cuando sea posible), el código fuente abierto o cursos educativos abiertos entre otros (5).

El presente año 2019 va camino de convertirse en un año importante en cuanto al acceso abierto, ciencia abierta se refiere. Están confluyendo una serie de circunstancias (algunas nuevas y otras no tanto) que pueden favorecer un impulso importante en el modo de publicación científica y su relación con la ciencia abierta $(6,7)$, entre otras:

- La aparición de plataformas de publicación basadas en un modelo de investigación abierta, del tipo Wellcome Research.

- El rápido crecimiento y aceptación de repositorios de preprints, esto es, la versión del artículo sin pasar revisión por pares.

- La obligación de publicar en abierto los datos de investigación, lo que ha traído la aparición de grandes repositorios de datos.

- Presentación del Plan S.

- Aprobación de la Declaración de San Francisco sobre Evaluación de la Investigación.

- Cambios en las negociaciones de los grandes acuerdos nacionales con las principales editoriales científicas.

\section{EL PLAN S - SU RELACIÓN CON LA RUTA DORADA}

Uno de los cambios o novedades que trajo 2018 fue el Plan S, publicado a principios de septiembre de ese año.

El Plan S es una iniciativa de los financiadores de la investigación (inicialmente europeos), bajo el paraguas del cOAlition $S$ (consorcio internacional de financiadores de investigación). Está liderado por Robert Jan Smits, enviado especial para Acceso Abierto de la Comisión Europea y por 
Science Europe (Asociación de Organizaciones Financiadoras de Investigación -RFO- y de Organizaciones que desarrollan Investigación -RPO).

Su principal objetivo es impulsar el avance del acceso abierto inmediato en Europa a través de 10 principios que actualmente se están debatiendo en un plan de acción preliminar.

A pesar de ser una iniciativa europea que está centrada en agencias de financiación científica, su objetivo es lograr el mayor respaldo posible de la comunidad científica a nivel global (8).

La idea principal del Plan S es que a partir del 1 de enero de 2020 las publicaciones científicas que sean resultado de investigaciones que reciban ayudas procedentes de consejos de investigación y organismos de financiación nacionales y europeos, deben publicarse en revistas o plataformas de acceso abierto (9).

Y además establece 10 principios:

1. Los autores conservarán el copyright de sus publicaciones sin restricciones. Todas las publicaciones deben publicarse bajo una licencia de acceso abierto, preferentemente una licencia Creative Commons de tipo "by" (atribución). En cualquier caso, la licencia aplicada debe cumplir con los requisitos definidos por la Declaración de Berlín.

2. Los financiadores garantizarán el establecimiento de criterios y requisitos sólidos para los servicios que deben cumplir las revistas de acceso abierto de alta calidad y las plataformas de acceso abierto que sean compatibles con los requisitos establecidos en la Declaración de Berlín.

3. En caso de que todavía no existan revistas de acceso abierto o plataformas de alta calidad, los financiadores, de forma coordinada, proporcionarán incentivos para establecerlos y respaldarlos cuando sea apropiado; también proporcionarán el soporte necesario para implementar infraestructuras de acceso abierto cuando se necesite.

4. Cuando sea aplicable, las tarifas de publicación de acceso abierto han de estar cubiertas por los financiadores o universidades, no por investigadores individuales.

5. Cuando se apliquen las tarifas de publicación en acceso abierto, su financiación debe estar estandarizada y limitada (en toda Europa).

6. Los financiadores solicitarán a las universidades, organizaciones de investigación y bibliotecas alinear sus políticas y estrategias, en particular para garantizar la transparencia.

7. Los principios anteriores se aplicarán a todos los tipos de publicaciones académicas, pero se entiende que la línea de tiempo para lograr el acceso abierto para monografías y libros puede ser más larga que el 1 de enero de 2020.

8. Se reconoce la importancia de los archivos y repositorios abiertos para alojar los resultados de la investigación.

9. El modelo de publicación "híbrido", aquel en el que la revista mantiene de forma simultánea las tarifas de suscripción clásica y la posibilidad de publicar en OA, previo pago de unas tasas, para poner el artículo accesible desde el momento de su publicación, no cumple con los principios anteriores.

10.Los financiadores supervisarán tanto el cumplimiento como el procedimiento de sanción, si corresponde.

Las reacciones al Plan S han sido de lo más variadas y las críticas no son pocas: tanto de los editores (comerciales y sociedades científicas), como de los propios investigadores.

Preocupan, especialmente a las casas editoras, las repercusiones económicas que su aplicación pueda tener en sus modelos de negocio, la forma en la que se va a configurar el cambio (en cuanto a los tiempos o en la forma en que los países trasvasarán los cambios a su organización nacional) (8). 
Otra de las principales críticas al Plan es que no tiene en cuenta la diferente realidad en la forma de publicar en unas disciplinas y otras (en clara alusión al veto del Plan S a las revistas híbridas, especialmente importantes en disciplinas como la Química) (10)

Para algunos, además, los países y agencias participantes son insuficientes (11). Sin embargo, como puede consultarse en la página del Plan, las instituciones participantes son muchas y variadas y aunque son principalmente europeas, cuentan con el apoyo de instituciones financiadoras tan importantes como la Fundación Bill y Melinda Gates, la Wellcome Trust, National Institute for Health Research o la Marie Curie Alumni Association(12,13). Ninguna institución española, por el momento, se ha adherido al Plan.

También han vuelto a ponerse sobre la mesa algunas de las tradicionales críticas sobre la baja calidad y ausencia de una revisión por pares adecuada en las revistas OA, una oportunidad para las revistas depredadoras e, incluso, «poner fin a la cooperación científica global» (6). Sin embargo, la mayoría de las críticas no tienen un fundamento contrastado ni demostrado.

A pesar de todas estas críticas, estamos en un momento que puede suponer una oportunidad de cambio en la forma de publicar, difundir y acceder a la literatura científica. Es una situación que implica:

- Un reto para las agencias financiadoras, que han de apostar de forma clara por la publicación en abierto, introduciendo otros criterios para evaluar los trabajos (además del "tradicional" factor de impacto), tales como las altmetrías o la presencia de revistas en repositorios de acceso abierto como valor añadido de un trabajo.

- Una gran reto para las instituciones y sus investigadores, que podrán plantearse publicar en otras revistas teniendo en cuenta criterios diferentes al factor de impacto de la revista.

- Un conflicto para los intereses de las editoriales (especialmente aquellas que copan el mercado), que deberán cambiar su modelo de negocio e introducir la filosofía del acceso abierto de forma real.

\section{El apoyo e impulso a la Ciencia: conflictos con las editoriales}

Con el objetivo de obligar a las grandes editoriales a adoptar un modelo de acceso abierto, son ya muchos los países que han iniciado una serie de movimientos que serán clave para el futuro de la ciencia europea.

La aparición del Plan S y el gran impulso que la ciencia abierta está tomando en los últimos años es una consecuencia de la necesidad que tiene la comunidad científica de acceder a los resultados de la investigación en unas condiciones razonables. El problema que se viene arrastrando en los últimos años y que finalmente ha "explotado" es una conjunción de cuestiones económicas y legales.

Por un lado, las instituciones deben desembolsar grandes cantidades de dinero para que sus investigadores puedan acceder a los resultados de las investigaciones que publican en las revistas. Los investigadores deben publicar sus trabajos, preferiblemente en revistas de alto impacto para asegurarse una buena evaluación por parte de las agencias destinadas a tal efecto y obtener la necesaria financiación.

Por otro lado, en muchas ocasiones publicar en esa revistas supone ceder los derechos de explotación del trabajo, de modo que el investigador no podrá depositarlo en el repositorio de su institución o distribuirlo entre sus alumnos sin antes obtener el permiso de la editorial. Las posibilidades de negociar con la editorial por no perder esos derechos son poco realistas.

Si unimos el malestar de instituciones e investigadores, con las crisis económicas que hemos sufrido (primero en la década de los 90's y más recientemente en 2007 y años siguientes) y la rígida actitud de las grandes editoriales ante estos cambios, no es de extrañar que tanto el acceso abierto 
como la ciencia abierta cuenten cada vez con más apoyo a nivel internacional y que los grandes acuerdos con los editores peligren o estén en crisis (14).

Así, desde 2008 estamos asistiendo a lo que podríamos llamar una rebelión por parte de la comunidad científica a estos grandes grupos editoriales y, lo más importante, no se trata ya de iniciativas aisladas sino de decisiones apoyadas por las instituciones académicas de los diversos países que han dicho basta a estos grupos empresariales y sus condiciones.

De nuevo, fueron los países nórdicos (principales apoyos del Plan S) los que tomaron la iniciativa en este sentido, pero pronto se unieron instituciones de Norte América y Canadá. Veamos algunos ejemplos, aunque hay muchos más (15). Puede consultarse una secuencia completa ordenada cronológicamente desde 2008 en un artículo publicado en Sparc titulado "Big Deal Cancellation Tracking"(14).

\section{Alemania}

El 17 de julio de 2017 se publicó una noticia en la revista The Scientist en la que se anunciaba que las universidades alemanas habían decidido no renovar los contratos que estaban negociando con la editorial Elsevier, Springer Nature y Wiley una vez que acabasen (a finales de 2017). Para ello se creó el Projekt DEAL un consorcio de bibliotecas universitarias e institutos de investigación en Alemania, que negocia grandes acuerdos con editoriales de revistas electrónicas.

Se trató de una medida de presión para conseguir, en términos generales, un mejor precio en la nueva licencia nacional, y en términos particulares conseguir que las instituciones alemanas pagasen por artículos publicados y no por suscripción de revistas y que estas estuviesen disponibles de forma abierta e inmediata para todos los investigadores.

Los resultados en Alemania son bastante satisfactorios, ya que el 15 de enero de 2019, la propia página del consorcio anunciaba que habían un acuerdo con la editorial Wiley basado en el modelo "Publish\&Read". Este acuerdo de 3 años supone el logro de una de las principales reivindicaciones del proyecto que siempre ha sido conseguir la publicación de todos los futuros artículos científicos en Acceso Abierto, sin coste extra y el acceso permanente a todas las revistas electrónicas de la editorial desde 1997 para las más de 700 instituciones académicas participantes a la actualidad. Aunque las negociaciones con Springer siguen su curso y con Elsevier permanecen estancadas, no cabe duda de que Alemania ha resultado victoriosa y que los investigadores alemanes publicarán en condiciones más ventajosas (16).

\section{Holanda}

En 2017 la Asociación de Rectores de las Universidades de Holanda (VSNU) decidió cancelar sus suscripciones con Oxford University Press. Esta asociación está muy implicada en el acceso abierto y recientemente ha publicado el Roadmap Open Access 2018- 2020, para lograr que el $100 \%$ de sus publicaciones estén en abierto en 2020.El Gobierno holandés es, juntamente con el británico, el más implicado en conseguir que en 2020 todas las publicaciones científicas estén en acceso abierto, tal y como mostró mientras presidió la Unión Europea (a través de la publicación del informe Amsterdam Call) (17-20).

\section{Francia}

Han sido dos las instituciones francesas que durante 2018 han roto sus acuerdos con la editorial Springer Nature: Le Consortium Couperin y la Université de Lorraine (14). 
El consorcio Couperin (que engloba más de 250 instituciones de investigación de educación superior y de salud) se gastó 5 millones de euros en suscripciones con Springer.

El consorcio detectó un significativo descenso en el uso de las revistas y no consiguió acordar con Springer un descenso de las suscripciones, lo que le llevó a decidir la cancelación de las mismas.

La Université de Lorraine "sufrió" desde 2007 a 2017 un aumento del 60\% en el precio de las suscripciones a revistas de Springer, lo que ha conllevó que la universidad se replantease el modelo de acceso a las publicaciones científicas, enfocándose hacia un modelo más abierto y transparente.

La consecuencia fue que en 2018 decidieron cancelar las suscripciones que tenían suscritas con Springer Nature y con parte del dinero ahorrado decidieron implementar nuevas iniciativas de publicación más acordes con el acceso abierto, la transparencia y un modelo centrado en la comunidad científica, tales como OpenEdition, Erudit, Open Library of Humanities, Sci-Post, o la editorial EDP Sciences.

\section{Universidad de California (EE.UU.)}

En marzo de 2019 la Universidad de California publicaba un comunicado en el que explicaba que cancelaba sus suscripciones con la editorial Elsevier debido a los costes de las revistas y a favor del acceso abierto (21). Fue la propia presidenta de la Universidad, Janet Napolitano (Secretaria de Seguridad Nacional en la Administración Obama), quien escribe una carta abierta el 28 de febrero de 20219 en la que explica la decisión (22).

La decisión de la Universidad de California es el resultado de 8 meses de negociaciones con la editorial Elsevier, tras haberse negado esta última a cerrar un acuerdo para reducir las cuotas de suscripción que la universidad tiene que pagar por acceder a las publicaciones y que haría que todos los artículos publicados por los investigadores de la universidad estuviesen disponibles en abierto de forma inmediata para todo el mundo.

No cabe duda de que es un varapalo para la editorial, ya que la Universidad de California pagaba alrededor de 11 millones de dólares al año por suscribir productos con Elsevier.

No obstante, este no es el fin del camino, ya que la propia Universidad reconoce que está dispuesta a retomar las negociaciones con Elsevier, tal y como ha manifestado en un comunicado del 20 de marzo a través de su oficina de comunicación académica (Office of Scholarly Communication) (23).

\section{Canadá}

Dos importantes instituciones académicas canadienses han manifestado públicamente su apoyo a la decisión tomada por la Universidad de California. Se trata de CRKN (Canadian Research Knowledge Network) y de CARL (Canadian Association of Research Libraries), quienes han publicado una noticia en la web de la primera en la que explican los motivos de manifestar públicamente su apoyo a la UC y se comprometen a trabajar conjuntamente y con otras instituciones canadienses para articular un "enfoque eficaz de hecho en Canadá para promover el acceso abierto" (24).

\section{Suecia}

En junio de 2018 el consorcio sueco Bibsam (formado por 85 universidades e institutos de investigación) decidió no renovar sus acuerdos con la editorial Elsevier al no lograr un entendimiento relacionado con la publicación en abierto. 
El gobierno sueco ha dado prioridad a que se produzca una transición de las suscripciones hacia el acceso abierto para 2026. Con este fin, el Consorcio Bibsam buscó negociar el acceso abierto inmediato a todos los artículos publicados en revistas Elsevier por investigadores afiliados a organizaciones participantes, así como un modelo de precios sostenible que permitiera una transición al acceso abierto.

Los investigadores suecos escriben alrededor de 4.000 artículos al año en revistas Elsevier y para poder publicar en abierto las instituciones suecas tuvieron que desembolsar, en 2017, 1,3 millones de euros, además de los 12 millones de euros en tarifas de suscripción, unas cifras relamerte insostenibles. Este ruptura supone que a partir de 2017 ya no hay acceso a revistas Elsevier pero sí se han mantenido el acceso al contenido más antiguo (de 1995 a 2017), según los términos de cese de la licencia $(14,17)$.

\section{Norvega}

Las instituciones de investigación noruegas han decidido no renovar sus contratos con Elsevier, según una noticia publicada en My New Desk el 12 de marzo de 2019 (25).

El gobierno noruego pretende que todos los artículos de investigación financiados con fondos públicos estén disponibles en abierto para 2024 y que se pase de pagar por leer artículos mediante acuerdos de suscripción a pagar por la publicación por artículos, pero con la condición de que éstos estén disponibles inmediatamente, a semejanza de las reivindicaciones que hizo en su momento Alemania.

Las negociaciones con Elsevier corren a cargo de Unit (dirección de TIC que comparte recursos de Educación Superior e Investigación) e incluyen a 44 instituciones miembros (universidades, colegios universitarios, institutos de investigación y hospitales).

Los investigadores suecos publican alrededor de 200 artículos a año en revistas de Elsevier y en 2018 las instituciones noruegas pagaron alrededor de 9 millones de euros en gastos por suscripción.

A pesar de que este año no se ha llegado a un acuerdo con la gran editorial para leer los nuevos artículos, los investigadores suecos sí podrán seguir publicando en revistas Elsevier como antes a la vez que podrán seguir consultando los artículos publicados en muchas revistas hasta 2018.

\section{Taiwán}

En 2017 el consorcio taiwanés CONCERT compuesto por más de 200 universidades nacionales, instituciones académicas y organizaciones gubernamentales, decidió cancelar sus contratos con Elsevier. CONCERT intentó conseguir precios de suscripción más bajos, así como una mayor flexibilidad para cancelar títulos concretos y al no llegar a acuerdo con Elsevier rompieron su acuerdo con la gran editorial. En la práctica esta situación ha supuesto que más del $75 \%$ de las bibliotecas de las universidades de Taiwan cancelaran su acceso a las revistas editadas por Elsevier.

\section{CONCLUSIONES}

Estamos en una época de grandes cambios y aunque es pronto para vislumbrar el recorrido que tendrán todas estas iniciativas a favor de la ciencia abierta y el acceso abierto, podemos extraer una serie de conclusiones: 
- Asistimos a una gran ventana de oportunidad para sumar nuevos agentes que se adhieran e impulsen el acceso abierto de la literatura científica.

- Los países están empezando a rebelarse contra el monopolio de las grandes editoriales (ya no son los únicos que marcan las reglas).

- Hay bastante desigualdad en las reacciones, por ejemplo, España no aparece en ninguna de estas iniciativas, aunque, en marzo de 2016 el Instituto de Salud Carlos III, organismo público financiador de la investigación biomédica en nuestro país, firmó la Declaración de Berlín. Frente a las grandes editoriales y aunque ha habido intentos de negociación de una licencia nacional, no se ha conseguido hacer mucha presión, quizás por la falta de unidad.

- Los grandes perjudicados son los investigadores (porque la cancelación de las suscripciones hace que tengan dificultades para acceder a trabajos que necesitan para desarrollar sus investigaciones);

- La resolución del conflicto dependerá, en buena medida, de cómo se organice en Europa este Plan y del apoyo por parte de las agencias evaluadoras nacionales, que tendrán que planteary buscar criterios alternativos para evaluar ciencia.

Una época de grandes cambios que no dejarán indiferente a nadie.

\section{BIBLIOGRAFÍA}

1. Suber P. Open Access. Cambridge, MA: The MIT Press; 2013.

2. Budapest Open Access Initiative (BOAI) [monografía en Internet]. BOAl; 2002 [consultada: 10/03/2019]Disponible en: http://bit.ly/2P8NrBx

3. Bethesda Statement on Open Access Publishing [monografía en Internet]. Bethesda; 2003[consultada: 10/03/2019]. Disponible en: http://bit.ly/2ligLVI

4. Comisión Europea. Informe anual sobre las actividades de investigación y desarrollo tecnológico de la Unión Europea y seguimiento de Horizonte 2020 en 2017 [monografía en Internet]. Bruselas: Comisión Europea; 2018 [consultada: 10/03/2019].Disponible en: http://bit.ly/2v4vxGY

5. Alessandroni N, Melero R, Rogel R, Vilches C, editores. Manual de Capacitación sobre Ciencia Abierta [monografía en Internet]. 2019 [consultada: 10/03/2019]. Disponible en: http://bit. Iy/2Z6JK49. DOI: 10.5281/zenodo.2583101

6. Velterop J. ¿Es inminente un impulso dramático al acceso abierto? ¡Creo que sí! [monografía en Internet]. 2019 [consultada: 15/03/2019]. Disponible en: http://bit.ly/2VHvWuE

7. KileyR. Wellcome Open Research: a summary of year 2 [entrada de Blog].2019 [consultada: 15/03/2019] Disponible en: http://bit.ly/2UzcRys

8. Bernal I. Plan S: Principios, hoja de ruta y reacciones. Enredadera.2018;32:18-20.

9. Plan-S: Making full and immediate Open Access a reality[página Web]. Bruselas: Science Europe; [consultada: 25/03/2019]. Disponible en: http://bit.ly/2lwR9DU

10. Plan S Open Letter -Reaction of Researchers to Plan S: Too Far, Too Risky [monografía en Internet]. 2018 [consultada: 15/03/2019]. Disponible en: http://bit.ly/2GladDz

11. Dal-Ré R. Plan S: Funders are committed to open access to scientific publication. Eur J Clin Invest. 2019: e13100. PMID: 30838638;DOI: 10.1111/eci.13100

12. What's Plan S, funders and supporters [página Web]. Bruselas: Science Europe; [consultada: 25/03/2019]. Disponible en: http://bit.ly/2V0oM7C 
13. Rabesandratana, T. Will the world embrace Plan $\mathrm{S}$, the radical proposal to mandate open access to science papers? [monografía en Internet]. 2019 [Consultada: 08/04/2019].Disponible en: http://bit.ly/2Giq1Ga

14. SPARC. Big Deal cancellation tracking [monografía en Internet]. [Consultada: 08/04/2019].Disponible en: http://bit.ly/2UXTTRi

15. Else H. Europe's open-access drive escalates as university stand-offs spread. Nature. 2018;557(7706):479-80. PMID: 29789729; DOI: 10.1038/d41586-018-05191-0

16. Projekt DEAL [página Web].Freiburg y Munich, Alemania: University Library Freiburg y Max Planck Digital Library Munich; [consultada: 08/04/2019].Disponible en: http://bit.ly/2P8MysO

17. Anglada L. El acceso abierto: pasos adelante, pasos atrás y conflictos con editoriales [entrada de Blog]. Barcelona: Blok de bid; 2018 [consultada: 11/04/2019]. Disponible en: http://bit. ly/2Go9lsB

18. No agreement between VSNU and Oxford University Press [monografía en Internet]. Leiden: Leiden University; 2017 [consultada: 11/04/2019]. Disponible en: http://bit.ly/2GgCMkr

19. Roadmap Open Access 2018 - 2020 [monografía en Internet]. La Haya: The Association of Universities in the Netherlands; 2018 [consultada: 11/04/2019]. Disponible en: http://bit.ly/2lkOgIV

20. Amsterdam Call for Action on Open Science [monografía en Internet]. Netherlands; 2016 [consultada 12/04/2019]. Disponible en: http://bit.ly/2GI7Q3M

21. Yirka B. University of California terminates subscriptions to Elsevier [entrada de Blog].California, USA: University of California; 2019 [consultada: 11/04/2019]. Disponible en: http://bit. Iy/2V2RsNC

22. UC Office of the President. UC terminates subscriptions with world's largest scientific publisher in push for open access to publicly funded research [monografía en Internet]. California, USA: University of California; 2019 [consultada: 11/04/2019]. Disponible en: http://bit.ly/2Gc8kl7

23. Office of Scholarly Communication. UC and Elsevier: Open Statement: Why UC Cut Ties with Elsevier[entrada de Blog]. California, USA: University of California; 2019 [consultada: 11/04/2019]. Disponible en: http://bit.ly/2UGMnLy

24. Canadian Research Knowledge Network (CRKN). CRKN and CARL Support the University of California in Taking a Bold Stand for Openly Available Research [monografía en Internet]. Ottawa, Canada: CRKN; 2019 [consultada: 11/04/2019]. Disponible en: http://bit.ly/2UxzO4S

25. UNIT Mynewsdesk. Norwegian research institutions have decided not to renew their agreement with Elsevier [monografía en Internet]. UNIT Mynewsdesk; 2019 [consultada: 11/04/2019].Disponible en: http://bit.ly/2UygCnl 